\title{
Urinary neutrophil gelatinase-associated lipocalin is a biomarker of delayed graft function after kidney transplantation
}

This article was published in the following Dove Press journal:

Transplant Research and Risk Management

20 January 2017

Number of times this article has been viewed

\author{
Irene Capelli \\ Olga Baraldi \\ Giorgia Comai \\ Elisa Sala \\ Maria Cappuccilli \\ Chiara Donadei \\ Vania Cuna \\ Maria Laura Angelini \\ Gabriele Donati \\ Gaetano La Manna \\ Department of Experimental \\ Diagnostic and Specialty Medicine \\ (DIMES), Nephrology, Dialysis and \\ Renal Transplant Unit, St Orsola \\ Hospital, University of Bologna, \\ Bologna, Italy
}

Background: Acute kidney injury occurring after kidney transplantation frequently leads to delayed graft function with detrimental long-term effects on graft survival. Neutrophil gelatinase-associated lipocalin (NGAL) has been validated as a biomarker for posttransplant acute kidney injury. This observational study aimed to assess the effectiveness of urinary NGAL as a predictive marker of delayed graft function.

Materials and methods: Forty-three consecutive patients who received renal transplant were included in the study. Urine samples were collected before transplant (if available) and at days $1,3,7,14$, and 30 after transplant, and urinary NGAL levels were quantified by enzyme-linked immunosorbent assay.

Results: Urinary NGAL progressively decreased after transplant in patients with both delayed and immediate graft function. However, urinary NGAL concentration remained significantly higher in the presence of delayed graft function in the first 14 days after transplant. The area under the receiver operating characteristic curve showed that the ability of urinary NGAL to predict delayed graft function was accurate at 1 st and 3rd days after transplant.

Conclusion: The relative decrease of urinary NGAL concentration rather than its absolute value may be relevant to predict delayed graft function after renal transplant. In particular, urinary NGAL area under the curve for 3 days seems to be a more valuable parameter of decision making in the early posttransplant period.

Keywords: area under the curve, delayed graft function, immediate graft function, kidney transplant, NGAL, acute kidney injury

\section{Introduction}

Acute kidney injury (AKI) occurring after renal transplantation frequently progresses to delayed graft function (DGF). Poor kidney function in the first week of graft life is detrimental to allograft longevity and may cause poor graft survival, acute rejection, and impaired renal function. ${ }^{1,2}$ In addition to DGF, even mild to moderate posttransplant graft dysfunction, namely, slow graft functions, has been proven to affect long-term graft survival.,4 DGF prevalence is constantly growing, mostly due to the use of organs recovered from expanded criteria donors and prolonged cold ischemia times. $^{5-7}$ Validation of immune or nonimmune biomarkers potentially able to immediately predict AKI after transplant could improve the possibility of a timely diagnosis and reduce the risk of DGF. ${ }^{8}$

In the last few years, great interest has been focused on neutrophil gelatinaseassociated lipocalin (NGAL), a promising biomarker for the prediction of kidney
Correspondence: Gaetano La Manna Department of Experimental, Diagnostic and Specialty Medicine (DIMES) -

Nephrology, Dialysis and Transplantation Unit, St Orsola Hospital, University of Bologna, Via G. Massarenti 9 (Pad. 15), 40138 Bologna, Italy

Tel +3905 I 2144577

Fax +39051344439

Email gaetano.lamanna@unibo.it
Transplant Research and Risk Management 2017:9 I5-2I 
injury in the early posttransplant period. The advantage of NGAL lies in the fact that kidney epithelia express and excrete large quantities of NGAL into urine immediately after ischemia-reperfusion injury, anticipating the rise of serum creatinine by several hours. It has been reported that increased local production of NGAL by the tubular epithelium of DGF kidneys contributes to the high circulating and urine levels, reflecting the ischemia/reperfusion stress applied to the transplanted kidney before organ withdrawal, during the storage period, and during successive reperfusion. ${ }^{9-11}$

NGAL is a $25 \mathrm{kDa}$ protein belonging to the lipocalin superfamily. It was initially found in activated neutrophils; however, many other cells, like kidney tubular cells, may produce it in response to various insults. ${ }^{12}$

Both urinary NGAL and plasma NGAL have been extensively investigated as reliable biomarkers in several clinical conditions, including cardiac surgery-related AKI, poor renal function in very low birth weight infants, critically ill patients, and DGF in kidney transplantation. ${ }^{13-16}$ In addition, we have recently described the ability of NGAL to induce immune tolerance by upregulating human leukocyte antigen (HLA-G) expression and expansion of T-regulatory cells in healthy donors, providing the basis for further studies to evaluate its possible role in immunomodulation and tolerance induction in transplant recipients. ${ }^{17}$

This mounting evidence led to evaluate the role of NGAL after kidney transplant. Plasma levels of NGAL rapidly decrease when the renal function is restored. This may be due to lower NGAL production, negative balance between extrarenal NGAL synthesis and renal washout, or renal uptake modification. NGAL is physiologically filtered by the glomerulus and almost completely reabsorbed in the proximal tubule. ${ }^{14}$ After ischemia-reperfusion injury in transplanted kidneys, tubular reabsorption may still be impaired even in the presence of normal glomerular filtration rate (GFR) and immediate graft function (IGF). Therefore, it is crucial to promptly discriminate early from DGF. To this purpose, here, we propose a prospective observational study aimed to assess the effectiveness of urinary NGAL as a predictive marker of DGF after kidney transplantation.

\section{Materials and methods Study design and patients}

This is a prospective observational study on 43 consecutive patients who received renal transplantation from deceased donors at our Kidney Transplant Center from February 2009 to May 2010.
All of them were treated with the same immunosuppressive therapy based on steroids (methylprednisolone $500 \mathrm{mg}$ at day $0,250 \mathrm{mg}$ at day $1,125 \mathrm{mg}$ at day $2,80 \mathrm{mg}$ at day 3 , and tapered until the final level of $5 \mathrm{mg}$ per day was reached), tacrolimus (5-8 $\mathrm{ng} / \mathrm{mL})$, and mycophenolic acid (1440 mg until day 14 , then $720 \mathrm{mg}$ ). The following demographic, clinical, and transplant-related characteristics of all the patients were collected: sex, recipient age, donor age, cold ischemia interval, number of HLA mismatches, time on dialysis prior to transplantation, dialysis modality, primary renal disease, and incidence of posttransplant complications.

At 2 years after transplantation, estimated GFR (eGFR) was calculated using the Chronic Kidney Disease Epidemiology Collaboration (CKD-EPI) equation, as a follow-up routine analysis.

To assess the predictive value of urinary NGAL in predicting DGF, the renal transplant recipients included in the study were divided into 2 groups: patients with DGF (DGF group, $n=18$ ) versus patients with IGF (IGF group, $n=25$ ). DGF was defined as the need for dialysis within the first 7 days after transplantation.

The study protocol was approved by the Independent Ethics Committee of the University Hospital of Bologna "S. Orsola-Malpighi", and a written informed consent was obtained from all the patients. The study was conducted according to the Helsinki Declaration.

\section{Urine collection and enzyme-linked immunosorbent assay (ELISA)}

Urine samples were collected before transplantation (if available) and at day 1, 3, 7, 14, and 30 after transplantation (T0, $\mathrm{T} 1, \mathrm{~T} 2, \mathrm{~T} 3, \mathrm{~T} 4$, and $\mathrm{T} 5$, respectively). Urine samples were centrifuged at $1500 \mathrm{rpm}$ for $10 \mathrm{~min}$, and the supernatants were stored at $-20^{\circ} \mathrm{C}$ until analysis.

All the samples were analyzed in the laboratory of our unit. Urinary levels of NGAL were assayed using a commercially available ELISA test, according to manufacturer's instructions (NGAL ELISA Kit 036; BioPorto Diagnostics, Grusbakken, Denmark). Briefly, the assay was performed on microplates coated with monoclonal antibody against human NGAL. Samples and calibrators were quantified in the same microplate. Urinary NGAL was detected with a colorimetric reaction by measuring the absorbance at $450 \mathrm{~nm}$ and its concentration was derived from the calibration curve and expressed in $\mathrm{ng} / \mathrm{mL}$. The sensitivity limit (minimum detectable concentration) was $1.4 \mathrm{pg} / \mathrm{mL}$. Intra-assay variation was determined by measurement of NGAL in 2 urine samples with 6-8 replicates, and the coefficient of variation was as follows: urine A $14 \%$ and urine B $7 \%$. Interassay variation 
was determined by measurement of NGAL in 2 diluted urine samples with 2-8 replicates in 2-4 separate assays. The following results were obtained: urine A $4 \%$ and urine B $8 \%$.

\section{Statistical analysis}

Continuous variables are presented as mean \pm standard deviation if normally distributed or median with interquartile range if non-normally distributed. Categorical variables are given as number and percentage. The normal distribution of each continuous variable was verified by the Kolmogorov-Smirnov test. Patients' characteristics were compared according to DGF or IGF (DGF group vs IGF group) using Student's $t$-test or Mann-Whitney test for continuous variables and chi-square test or Fisher's exact test for categorical variables. Urinary NGAL concentrations at each time point were compared between the DGF and IGF groups by Mann-Whitney test. Receiver operating characteristic (ROC) analysis was performed to estimate the accuracy of urinary NGAL to predict DGF or eGFR $\geq 60$ $\mathrm{mL} / \mathrm{min}$. The area under the ROC curve (AUC) was calculated with $95 \%$ confidence interval (CI) for patients with complete data at T1, T2, T3, and T4. The level of significance was set at $p<0.05$. Statistical analyses were carried out using SAS software, version 9.3 (SAS Institute, Cary, NC, USA).

\section{Results}

\section{Demographic, clinical, and transplant-related parameters of renal transplant recipients}

From February 2009 to May 2010, 44 consecutive patients who received kidney transplantation from deceased donors were enrolled in the study. Before transplantation, all patients were under chronic dialysis treatment. One patient who experienced acute rejection after 7 days from transplant was excluded from the analysis. In the overall transplant recipient population, 18 patients had DGF (DGF group) and 25 patients had immediate recovery of graft function (IGF group). The demographic, clinical, and transplant-related features of the 2 groups are summarized in Table 1.

Age of the patients ranged from 32 to 70 years, with no significant differences found between DGF group and IGF group. Likewise, the patients with DGF or IGF did not differ significantly in terms of donor age, cold ischemia interval, and number of HLA mismatches.

The renal transplant recipients with DGF had a longer dialysis vintage prior to transplantation as compared with those who had an immediate recovery of graft function ( $p=0.0204)$.

All the 18 patients in the DGF group had been under hemodialysis treatment before undergoing renal transplant,
Table I Demographic and clinical characteristics of patients

\begin{tabular}{|c|c|c|c|}
\hline Parameters & $\begin{array}{l}\text { Total } \\
\text { patients, } \\
\mathrm{N}=43(\%)\end{array}$ & $\begin{array}{l}\text { DGF } \\
\text { group, } \\
n=18(\%)\end{array}$ & $\begin{array}{l}\text { IGF } \\
\text { group, } \\
\mathbf{n}=25 \text { (\%) }\end{array}$ \\
\hline \multicolumn{4}{|l|}{ Sex } \\
\hline Male & $29(67.4)$ & II (6I.I) & $18(72)$ \\
\hline Female & $14(32.6)$ & $7(38.9)$ & $7(28)$ \\
\hline Recipient age, years & $54.0 \pm 9.6$ & $54.0 \pm 8.5$ & $54.0 \pm 10.5$ \\
\hline Donor age, years & $52.0 \pm 7.9$ & $53.2 \pm 8.1$ & $49.9 \pm 12.8$ \\
\hline Cold ischemia time, hours & $16.8 \pm 6.2$ & $\mid 7.1 \pm 7.3$ & $16.5 \pm 8.1$ \\
\hline $\begin{array}{l}\text { HLA-A, HLA-B, and HLA-DR } \\
\text { mismatches }\end{array}$ & $3.3 \pm 1.2$ & $3.4 \pm 1.1$ & $3.2 \pm 1.0$ \\
\hline $\begin{array}{l}\text { Time on dialysis before } \\
\text { transplant, months }\end{array}$ & $63.5 \pm 29.4$ & $75.6 \pm 25.2$ & $54.8 \pm 29.6^{*}$ \\
\hline \multicolumn{4}{|l|}{ Dialysis modality } \\
\hline Hemodialysis & $36(83.7)$ & $18(100)$ & $18(72)^{*}$ \\
\hline Peritoneal dialysis & $7(16.3)$ & $0(0)$ & $7(28)^{*}$ \\
\hline Presence of residual diuresis & $16(37.2)$ & $6(33.3)$ & $10(40)$ \\
\hline \multicolumn{4}{|l|}{ Primary nephropathy } \\
\hline Unknown etiology ESRD & $14(32.6)$ & $7(38.8)$ & $7(28)$ \\
\hline Glomerulonephritis & $13(30.3)$ & $4(22.2)$ & $9(36)$ \\
\hline APKD & $8(18.6)$ & $4(22.2)$ & $4(16)$ \\
\hline Cortical or tubular necrosis & I (2.3) & $\mathrm{I}(5.6)$ & $0(0)$ \\
\hline Interstitial nephritis & $3(7)$ & $0(0)$ & $3(12)$ \\
\hline Nephroangiosclerosis & $3(7)$ & $\mathrm{I}(5.6)$ & $2(8)$ \\
\hline Diabetic nephropathy & I (2.3) & $\mathrm{I}(5.6)$ & $0(0)$ \\
\hline Length of stay in hospital, days & $20.8 \pm 7.8$ & $18.0 \pm 6.2$ & $22.2 \pm 10.0$ \\
\hline \multicolumn{4}{|l|}{ Complication rate } \\
\hline Hemorrhage & $4(9.3)$ & I (5.6) & $3(12.0)^{*}$ \\
\hline Urinary fistula & I (2.3) & $0(0)$ & I (4.0) \\
\hline Abdominal surgery & I (2.3) & $\mathrm{I}(5.6)$ & $0(0)$ \\
\hline None & $32(74.4)$ & II (6I.I) & $21(84.0)$ \\
\hline
\end{tabular}

Notes: Continuous variables are presented as mean \pm standard deviation, and categorical variables as number (percentage). DGF group and IGF group were compared using Student's $t$-test for continuous variables and chi-square test for categorical variables. $* p<0.05$.

Abbreviations: APKD, adult polycystic kidney disease; DGF, delayed graft function; ESRD, end-stage renal disease; IGF, immediate graft function; HLA, human leukocyte antigen.

whereas 7/25 patients in the IGF group had been under peritoneal dialysis. So, chi-square test revealed a significant difference in the distribution of dialysis modality between the 2 groups $(p=0.0303)$.

In the overall patient population, end-stage renal disease, glomerulonephritides (mostly IgA nephropathy), and adult polycystic kidney disease were the most frequent primary nephropathies. Adult polycystic kidney disease (APDK) was more frequent in patients with DGF, while glomerulonephritis was more common in the IGF group, although the differences were not statistically significant. The length of hospital stay was similar in the 2 groups, with no graft rejection episodes occurring until the 30th day of follow-up.

Most of the transplant recipients included in the study had no posttransplant complications. Hemorrhage occurred more frequently in patients with IGF than in those with DGF (difference not significant). One patient in the IGF 
group had urinary fistula and 1 in the DGF group needed abdominal surgery.

\section{NGAL measurements}

Table 2 shows the urinary NGAL levels in patients in DGF group and IGF group over time (on the day before transplantation and at days 1, 3, 7, 14, and 30 after transplantation, indicated as T0, T1, T2, T3, T4, and T5, respectively). Since the data were non-normally distributed, the values are given as median with interquartile range.

Urinary NGAL concentrations progressively decreased after transplant in both groups of renal transplant recipients. However, in patients with IGF, NGAL levels showed a more rapid decrease within 3 days after transplantation. In DGF patients, NGAL concentration remained significantly higher at 14 days after transplantation, and then progressively decreased until the 30th day.

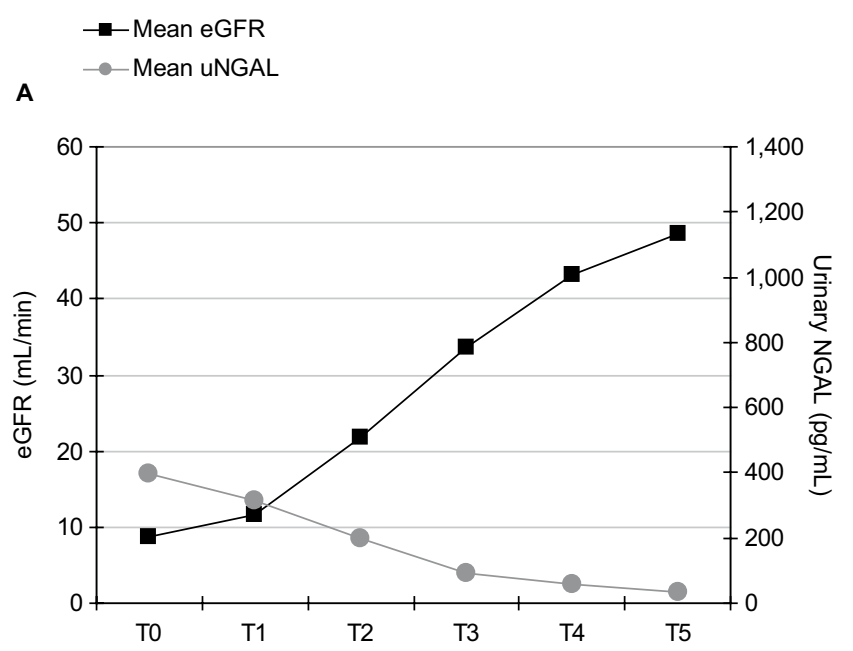

Table 2 Urinary neutrophil gelatinase-associated lipocalin in DGF group versus IGF group

\begin{tabular}{|c|c|c|c|}
\hline & DGF group, $n=18$ & IGF group, $n=25$ & $p$-Value \\
\hline T0 & $\begin{array}{l}380.7 \mathrm{pg} / \mathrm{mL}(280.5-396.9) \\
\mathrm{n}=6\end{array}$ & $\begin{array}{l}684.2(302.1-969.4), \\
n=10\end{array}$ & ns \\
\hline TI & $\begin{array}{l}594.2 \mathrm{pg} / \mathrm{mL}(295.4-12 \mid 2.2), \\
\mathrm{n}=12\end{array}$ & $\begin{array}{l}289.2(71.9-556.1) \\
n=23\end{array}$ & ns \\
\hline $\mathrm{T} 2$ & $\begin{array}{l}491.1 \mathrm{pg} / \mathrm{mL}(|97 .|-8 \mid 7.8) \\
\mathrm{n}=16\end{array}$ & $\begin{array}{l}107.6(44.5-197.7) \\
n=22\end{array}$ & 0.0022 \\
\hline T3 & $\begin{array}{l}227.8 \mathrm{pg} / \mathrm{mL}(\mathrm{I} \mid 8.9-472.8) \\
\mathrm{n}=16\end{array}$ & $\begin{array}{l}63.8(31.1-96.5), \\
n=20\end{array}$ & 0.0025 \\
\hline $\mathrm{T} 4$ & $\begin{array}{l}105.6 \mathrm{pg} / \mathrm{mL}(33.6-299.4) \\
\mathrm{n}=15\end{array}$ & $\begin{array}{l}33.4(21.0-60.0) \\
n=19\end{array}$ & 0.0244 \\
\hline T5 & $\begin{array}{l}31.6 \mathrm{pg} / \mathrm{mL}(|8.6-1| 1.2), \\
\mathrm{n}=13\end{array}$ & $\begin{array}{l}55.7(20.5-140.4) \\
n=16\end{array}$ & ns \\
\hline
\end{tabular}

Notes: Data are presented as median with IQR. DGF group and IGF group were compared using Mann-Whitney test. T0, day before transplant; TI, day I after transplant; T2, day 3 after transplant; T3, day 7 after transplant; T4, day 14 after transplant; T5, day 30 after transplant.

Abbreviations: DGF, delayed graft function; IGF, immediate graft function; IQR, interquartile range; ns, not significant.
B

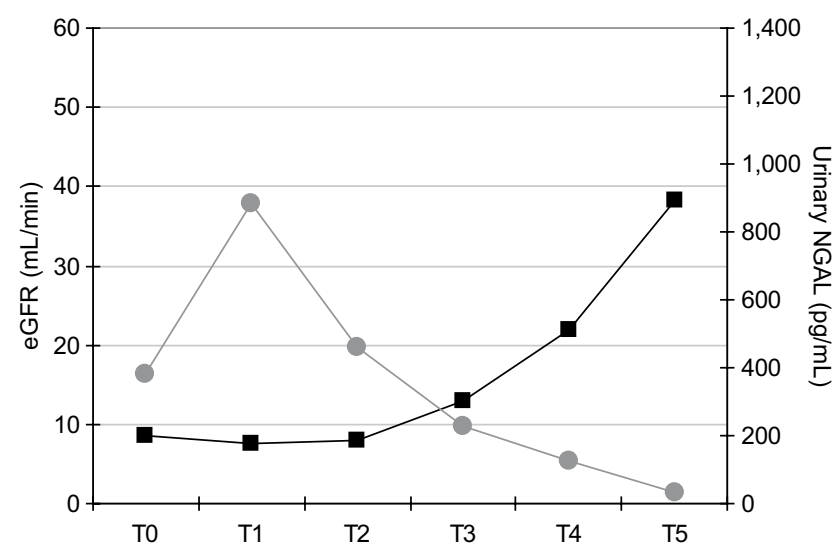

C

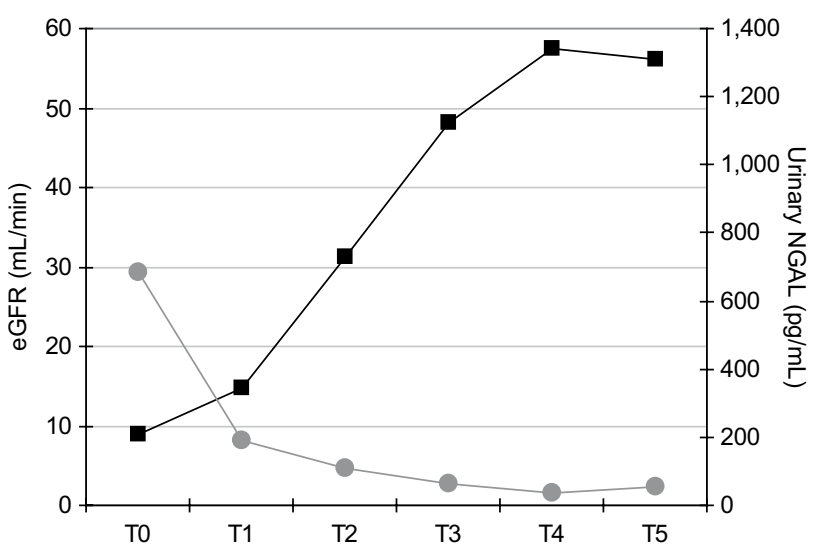

Figure I Mean eGFR values and median levels of urinary NGAL at T0, TI, T2, T3, T4, and T5 in the overall population (A), in patients with DGF (B) and in those with IGF (C). Note: T0, day before transplant; TI, day I after transplant; T2, day 3 after transplant; T3, day 7 after transplant; T4, day 14 after transplant; T5, day 30 after transplant. Abbreviations: DGF, delayed graft function; eGFR, estimated glomerular filtration rate (Chronic Kidney Disease Epidemiology Collaboration [CKD-EPI] equation); IGF, immediate graft function; NGAL, neutrophil gelatinase-associated lipocalin; uNGAL, urinary NGAL. 
A

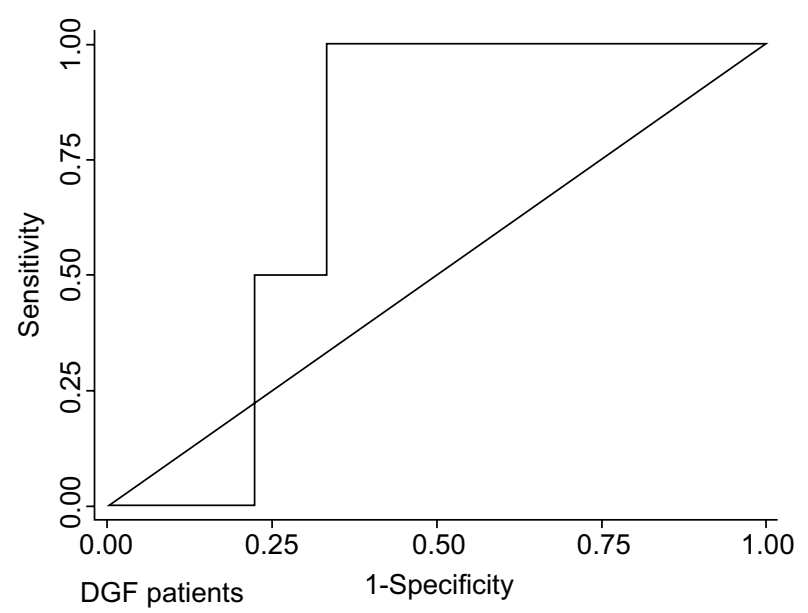

B

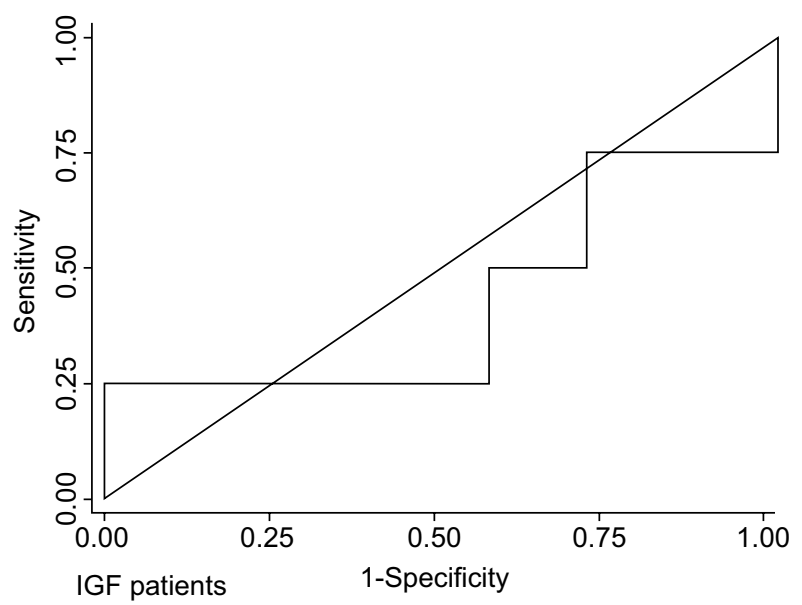

Figure 2 Cumulative area under the ROC curve for urinary neutrophil gelatinase-associated lipocalin levels measured at TI, T2, T3, T4 and T5 (day before transplant, and and days I, 3, 7, I4, and 30 after transplant), for predicting eGFR $\geq 60 \mathrm{~mL} / \mathrm{min}$. (A) DGF group ( $\mathrm{n}=18 ; \mathrm{AUC}=0.72 ; 95 \% \mathrm{Cl}$ : 0.4I-I.00); (B) IGF group ( $\mathrm{n}=25 ; \mathrm{AUC}=0.43$; 95\% Cl: 0.00-0.86).

Note: T0, day before transplant; TI, day I after transplant; T2, day 3 after transplant; T3, day 7 after transplant; T4, day I4 after transplant; T5, day 30 after transplant. Abbreviations: AUC, area under the curve; Cl, confidence interval; DGF, delayed graft function; eGFR, estimated glomerular filtration rate (Chronic Kidney Disease Epidemiology Collaboration [CKD-EPI] equation); IGF, immediate graft function; ROC, receiver operating characteristic.

The AUC showed that the ability of urinary NGAL to predict DGF was accurate at the 1st (AUC $=0.70 ; 95 \%$ CI: $0.48-0.92), 3$ rd (AUC $=0.79 ; 95 \%$ CI: $0.58-0.99), 7$ th $(\mathrm{AUC}=0.78 ; 95 \%$ CI: $0.58-0.98)$, and 14th $(\mathrm{AUC}=0.72$; 95\% CI: 0.52-0.92) days after transplantation.

After 2 years of follow-up, eGFR was evaluated based on the CKD-EPI equation. Figure 1 reports the changes over time in the median levels of urinary NGAL and the mean eGFR in the overall population (Figure 1A), in the patients with DGF (Figure 1B), and in those with IGF (Figure 1C). Figure 2 shows the cumulative AUC for urinary NGAL levels in relation to eGFR: the cumulative urinary NGAL in the first 2 weeks after transplant was found to be able to predict eGFR $\geq 60 \mathrm{~mL} / \mathrm{min}$ in patients who experienced DGF.

\section{Discussion}

This prospective observational study reports the experience of a single Italian kidney transplant center in the investigation of urinary NGAL as a potential biomarker of DGF in renal transplant recipients. Our results showed a progressive decline in urinary NGAL concentration over time until the end of the 1-month observation period following transplantation, but the rate of the decrease was significantly slower in patients with DGF. The measurement of cumulative urinary NGAL concentration for 14 days during hospitalization was found to be a valuable predictor of DGF. Moreover, after 2 years from transplant, the AUC of urinary NGAL in relation to graft function indicated a relationship between NGAL concentration and eGFR $>60 \mathrm{~mL} / \mathrm{min}$ in those patients who had experienced DGF.

NGAL has been described as a promising biomarker in a variety of renal and nonrenal conditions, and is routinely used for early diagnosis, predicting disease severity and clinical outcomes, and therapeutic monitoring. ${ }^{14,18} \mathrm{~A}$ previous report from our group described the ability of serum and urinary NGAL to predict renal function in the first weeks of life of preterm infants with a very low birth weight. We found that urinary NGAL was significantly higher in infants who suffered impaired renal function than in healthy controls. ${ }^{15}$ In adults, NGAL is a biomarker for acute renal injury ${ }^{16}$ and it is useful to evaluate the risk of DGF after kidney transplantation. ${ }^{9,10}$

NGAL increase occurs rapidly after acute injury, and is detectable within a few hours, characterizing NGAL as a troponin-like molecule for an early and sensitive reflection of kidney function, better than serum creatinine. It has been reported that the kidney epithelia express and excrete large quantities of NGAL into urine following stress by acute injury, reaching up to 1000 -fold induction in the most severe cases; thus, normalization for urinary creatinine level is not mandatory. ${ }^{19}$

Among the variety of assays and platforms currently available for the measurement of NGAL in both urine and blood, the ones that may be applied for a diagnostic use should be rapid and able to provide the results in $<1$ 
hour. In this study, we have measured urinary NGAL using an ELISA test, which represents a suitable choice for research purposes owing to the high sensitivity and reliability of this technique. However, this approach is less applicable in routine clinical practice when an early and timely diagnosis is required, because it does not run on automated clinical chemistry analyzers and is more time consuming and expensive (about EUR 27/test) than the other available assays.

Most of the patients in our population showed elevated urinary NGAL levels before transplant, possibly related to the chronic inflammatory status established by the previous dialysis treatment. Although the role of NGAL in dialyzed patients is less clear, Malyszko et al showed that both urinary and serum NGAL are able to predict CKD progression and are related to residual renal function, type of renal replacement therapy, and inflammation. ${ }^{20} \mathrm{We}$ found similar levels of pretransplant urinary NGAL in patients with DGF and IGF, likely due to high variability in their clinical history and status. However, the curves of urinary NGAL reduction were different between the 2 groups. In IGF patients, the NGAL rapidly decreased to levels below $200 \mathrm{ng} / \mathrm{mL}$, showing the most prominent reduction within the first 3 days and then reaching a plateau. In DGF patients, urinary NGAL levels remained high for several days and dropped concomitantly to DGF resolution. Therefore, it appears that the rate of NGAL decrease, rather than its absolute value, seems to predict DGF, as previously reported. ${ }^{21}$ Furthermore, we demonstrated that the measurement of cumulative NGAL concentrations 1 month after transplant was able to predict poor GFR at 2 years of follow-up.

The main limitation of the study was the low number of patients enrolled. Further larger studies may better define the clinical and predictive role of urinary NGAL in DGF onset. In this study, we observed that an increased or constantly high NGAL concentration during early monitoring after kidney transplant may indicate an extended injury with worse prognosis. Therefore, it seems to be useful to follow NGAL levels at least 1 month after transplant to identify the patients who might potentially experience long-term complications of DGF, including longer length of hospital stay, higher incidence of acute rejection, and poorer long-term graft survival. ${ }^{1,2,22,23}$ A diagnostic test, such as urinary NGAL, that may predict DGF early could allow a better management of transplant patients in both short- and long-term settings.

\section{Acknowledgment}

The authors would like to acknowledge the essential contribution of Dr Elisa Carretta for her invaluable support in the statistical analysis.

\section{Disclosure}

The authors report no conflicts of interest in this work.

\section{References}

1. Siedlecki A, Irish W, Brennan DC. Delayed graft function in the kidney transplant. Am J Transplant. 2011;11(11):2279-2296.

2. Yarlagadda SG, Coca SG, Formica RN Jr, Poggio ED, Parikh CR. Association between delayed graft function and allograft and patient survival: a systematic review and meta-analysis. Nephrol Dial Transplant. 2009;24(3):1039-1047.

3. Humar A, Ramcharan T, Kandaswamy R, Gillingham K, Payne WD, Matas AJ. Risk factors for slow graft function after kidney transplants: a multivariate analysis. Clin Transplant. 2002;16(6):425-429.

4. Scolari MP, Cappuccilli ML, Lanci N, et al. Predictive factors in chronic allograft nephropathy. Transplant Proc. 2005;37(6):2482-2484.

5. Arnau A, Rodrigo E, Minambres E, et al. Prediction of kidney transplant outcome by donor quality scoring systems: expanded criteria donor and deceased donor score. Transplant Proc. 2012;44(9):2555-2557.

6. Gandolfini I, Buzio C, Zanelli P, et al. The kidney donor profile index (KDPI) of marginal donors allocated by standardized pretransplant donor biopsy assessment: distribution and association with graft outcomes. Am J Transplant. 2014;14(11):2515-2525.

7. La Manna G, Conte D, Cappuccilli ML, et al. An in vivo autotransplant model of renal preservation: cold storage versus machine perfusion in the prevention of ischemia/reperfusion injury. Artif Organs. 2009; 33(7):565-570.

8. Halawa A. The early diagnosis of acute renal graft dysfunction: a challenge we face. The role of novel biomarkers. Ann Transplant. 2011;16(1):90-98.

9. Parikh CR, Jani A, Mishra J, et al. Urine NGAL and IL-18 are predictive biomarkers for delayed graft function following kidney transplantation. Am J Transplant. 2006;6(7):1639-1645.

10. Hall IE, Yarlagadda SG, Coca SG, et al. IL-18 and urinary NGAL predict dialysis and graft recovery after kidney transplantation. $J \mathrm{Am}$ Soc Nephrol. 2010;21(1):189-197.

11. Reese PP, Hall IE, Weng FL, et al. Associations between deceaseddonor urine injury biomarkers and kidney transplant outcomes. $J \mathrm{Am}$ Soc Nephrol. 2016;27(5):1534-1543.

12. Mishra J, Ma Q, Prada A, et al. Identification of neutrophil gelatinaseassociated lipocalin as a novel early urinary biomarker for ischemic renal injury. J Am Soc Nephrol. 2003;14(10):2534-2543.

13. Soni SS, Cruz D, Bobek I, et al. NGAL: a biomarker of acute kidney injury and other systemic conditions. Int Urol Nephrol. 2010;42(1):141-150.

14. Singer E, Marko L, Paragas N, et al. Neutrophil gelatinase-associated lipocalin: pathophysiology and clinical applications. Acta Physiol (Oxf). 2013;207(4):663-672.

15. La Manna G, Galletti S, Capelli I, et al. Urinary neutrophil gelatinaseassociated lipocalin at birth predicts early renal function in very low birth weight infants. Pediatr Res. 2011;70(4):379-383.

16. Haase-Fielitz A, Haase M, Devarajan P. Neutrophil gelatinase-associated lipocalin as a biomarker of acute kidney injury: a critical evaluation of current status. Ann Clin Biochem. 2014;51(Pt 3):335-351.

17. La Manna G, Ghinatti G, Tazzari PL, et al. Neutrophil gelatinaseassociated lipocalin increases HLA-G(+)/FoxP3(+) T-regulatory cell population in an in vitro model of PBMC. PLoS One. 2014;9(2): e89497. 
18. Devarajan P. Neutrophil gelatinase-associated lipocalin (NGAL): a new marker of kidney disease. Scand J Clin Lab Invest Suppl. 2008;241: 89-94.

19. Mori K, Nakao K. Neutrophil gelatinase-associated lipocalin as the real-time indicator of active kidney damage. Kidney Int. 2007;71(10): 967-970.

20. Malyszko J, Malyszko JS, Koc-Zorawska E, Kozminski P, Mysliwiec M. Neutrophil gelatinase-associated lipocalin in dialyzed patients is related to residual renal function, type of renal replacement therapy and inflammation. Kidney Blood Press Res. 2009;32(6):464-469.
21. Kanter J, Beltran S, Molina D, et al. Urinary neutrophil gelatinaseassociated lipocalin after kidney transplantation: is it a good biomarker to assess delayed graft function? Transplant Proc. 2013;45(4): 1368-1370.

22. Devarajan P. Neutrophil gelatinase-associated lipocalin (NGAL): a new marker of kidney disease. Scand J Clin Lab Invest Suppl. 2008; 241:89-94.

23. Rosenthal JT, Danovitch GM, Wilkinson A, Ettenger RB. The high cost of delayed graft function in cadaveric renal transplantation. Transplantation. 1991;51(5):1115-1118. 